\title{
OPERATORS WITH SINGULAR CONTINUOUS SPECTRUM: II. RANK ONE OPERATORS
}

\author{
R. DEL Rio ${ }^{1,3}$, N. MAKAROV ${ }^{2}$ AND B. Simon 3 \\ Division of Physics, Astronomy and Mathematics \\ California Institute of Technology, 253-37 \\ Pasadena, CA 91125
}

\begin{abstract}
For an operator, $A$, with cyclic vector $\varphi$, we study $A+\lambda P$ where $P$ is the rank one projection onto multiples of $\varphi$. If $[\alpha, \beta] \subset \operatorname{spec}(A)$ and $A$ has no a.c. spectrum, we prove that $A+\lambda P$ has purely singular continuous spectrum on $(\alpha, \beta)$ for a dense $G_{\delta}$ of $\lambda$ 's.
\end{abstract}

\section{$\S 1$. Introduction}

The subject of rank one perturbations of self-adjoint operators and the closely related issue of the boundary condition dependence of Sturm-Liouville operators on $[0, \infty)$ has a long history. We're interested here in the connection with BorelStieltjes transforms of measures $(\operatorname{Im} z>0)$ :

$$
F(z)=\int \frac{d \rho(x)}{x-z}
$$

where $\rho$ is a measure with

$$
\int(|x|+1)^{-1} d \rho(x)<\infty .
$$

In two fundamental papers Aronszajn [1] and Donoghue [5] related $F$ to spectral theory with important later input by Simon-Wolff [13]. In all three works, as in ours, the function ( $y$ real)

$$
G(y)=\int \frac{d \rho(x)}{(x-y)^{2}}
$$

plays an important role. Note we define $G$ to be $+\infty$ if the integral diverges. Note too if $G(y)<\infty$, then the integral defining $F$ is finite at $z=y$ and so we can and will talk about $F(y)$.

\footnotetext{
${ }^{1}$ Permanent address: IIMAS-UNAM, Apdo. Postal 20-726, Admon. No. 20, 01000 Mexico D.F., Mexico. Research partially supported by DGAPA-UNAM and CONACYT.

${ }^{2}$ This material is based upon work supported by the National Science Foundation under Grant No. DMS-9207071. The Government has certain rights in this material.

${ }^{3}$ This material is based upon work supported by the National Science Foundation under Grant No. DMS-9101715. The Government has certain rights in this material.

To appear in Commun. Math. Phys.
} 
Donoghue studied the situation

$$
A_{\lambda}=A_{0}+\lambda P
$$

where $P \psi=(\varphi, \psi) \varphi$ with $\varphi$ a unit vector cyclic for $A . d \rho$ is then taken to be spectral measure for $\varphi$, that is,

$$
\left(\varphi, e^{i s A_{0}} \varphi\right)=\int e^{i s x} d \rho(x)
$$

Aronszajn studied the situation

$$
H_{\text {formal }}=-\frac{d^{2}}{d x^{2}}+V(x)
$$

on $[0, \infty)$ where $V$ is such that the operator is limit point at $\infty$. Then, there is a one-parameter family of operators, $H_{\theta}$, with boundary condition

$$
u(0) \cos \theta+u^{\prime}(0) \sin \theta=0
$$

$\rho$ is the conventional Weyl-Titchmarsh-Kodaira spectral measure for a fixed boundary condition, $\theta_{0}$.

An important result of the Aronszajn-Donoghue theory is

Theorem 1. $E$ is an eigenvalue of $A_{\lambda}\left(\right.$ resp. $\left.H_{\theta}\right)$ if and only if

(i) $G(E)<\infty$

(ii) $F(E)=-\lambda^{-1} \quad\left(\right.$ resp. $\left.\cot \left(\theta-\theta_{0}\right)\right)$.

Our goal here is to the prove the following pair of theorems:

Theorem 2. $\{E \mid G(E)=\infty\}$ is a dense $G_{\delta}$ in $\operatorname{spec}\left(A_{0}\right)$ (resp. $\left.H_{\theta_{0}}\right)$.

Theorem 3. $\left\{\lambda \mid A_{\lambda}\right.$ has no eigenvalues in $\left.\operatorname{spec}\left(A_{0}\right)\right\}$ (resp. $\left\{\theta \mid H_{\theta}\right.$ has no eigenvalues in $\operatorname{spec}\left(H_{\theta_{0}}\right\}$ is a dense $G_{\delta}$ in $\mathrm{R}$ (resp. $\left.\left.[0,2 \pi]\right)\right)$.

While not stated precisely in those terms, Thm. 2 is a generalization of del Rio [4]. Gordon [8,9] has independently obtained these results by different methods.

Thm. 2 is quite easy and appears in $\S 2$. Thm. 3 is deeper and depends on some subtle estimates of $F$ found in $\S 3$ and applied in $\S 4$ to prove Thm. 3 .

The interesting applications of Thm. 3 found in $\S 5$ concern singular continuous spectrum. For example, suppose $A_{0}$ has spectrum $[0,1]$ but has no a.c. spectrum. By general principles, $A_{\lambda}$ has no a.c. spectrum either. Then, Thm. 3 says that for a dense $G_{\delta}$ of $\lambda, A_{\lambda}$ has purely singular continuous spectrum. This is especially interesting because there are examples where the Simon-Wolff theory implies that for Lebesgue a.e. $\lambda, A_{\lambda}$ has only point spectrum. However, this is not always the case. There exist $A_{0}$ and $P$ so that $\operatorname{spec}\left(A_{0}+\lambda P\right)$ is purely singular continuous on $(0,1)$ for all $\lambda$. However, Thm. 3 implies that it cannot happen that the spectrum is always pure point. 


\section{$\S 2$. Forbidden Energies}

In this section we'll essentially prove Thm. 2 .

Theorem 2.1. Let $d \rho$ be a measure obeying (1.2). Let

$$
G(y)=\int \frac{d \rho(x)}{(x-y)^{2}}
$$

Then, $\{y \mid G(y)=\infty\}$ is a dense $G_{\delta}$ in $\operatorname{supp}(d \rho)$, the support of $d \rho$.

Remarks 1. By the Thm. 1, only E's with $G(E)<\infty$ can be eigenvalues of $A_{\lambda}$ or $H_{\theta}$, so $E$ 's with $G(y)=\infty$ are "forbidden energies," that is, energies which cannot be eigenvalues.

2. If $\operatorname{supp}(d \rho)(=\operatorname{spec}(A))$ is perfect (no isolated points), then the theorem says the forbidden energies are locally uncountable in $\operatorname{supp}(d \rho)$.

3. Obviously, $\{y \mid G(y)=\infty\} \subset \operatorname{supp}(d \rho)$.

4. This says that $\{y \in \operatorname{supp}(d \rho) \mid G(y)<\infty\}$ has interior empty in $\operatorname{supp}(d \rho)$. Even more so is the interior empty in R. The theorem is a stronger result than interior empty in $\mathrm{R}$ since $\operatorname{supp}(d \rho)$ might itself have interior empty in $\mathrm{R}$.

Proof. The following are fundamental facts about Borel-Stieltjes transforms and their relation to $d \rho$ (see [3]):

(1) $\lim _{\epsilon \downarrow 0} F(E+i \epsilon) \equiv F(E+i 0)$ exists and is finite for Lebesgue a.e. $E$.

(2) $d \rho_{\mathrm{ac}}$ is supported on $\{E \mid \operatorname{Im} F(E+i 0)>0\}$.

(3) $d \rho_{\text {sing }}$ is supported on $\left\{E \mid \lim _{\epsilon \downarrow 0} \operatorname{Im} F(E+i 0)=\infty\right\}$.

If $G(y)<\infty$, it is easy to see that $\lim _{\epsilon \downarrow 0} F(E+i 0)$ exists is finite and real. Thus, if $G(y)<\infty$ on an interval $(\alpha, \beta) \subset \mathrm{R}$, then by (2), (3), $d \rho(\alpha, \beta)=0$, that is, $(\alpha, \beta) \cap \operatorname{supp}(d \rho)=\emptyset$. Thus, $\{y \mid G(y)=\infty\}$ is dense in $\operatorname{supp}(d \rho)$.

That $\{y \mid G(y)=\infty\}$ is a $G_{\delta}$ follows from the fact that $G$ is lower semicontinuous. To be explicit, let

$$
G_{m}(y)=\int \frac{d \rho(x)}{(x-y)^{2}+\left(m^{-1}\right)^{2}}
$$

which is a $C^{\infty}$ function by $(1.2)$ and $G(y)=\sup _{m} G_{m}(y)$. Thus

$$
\begin{aligned}
\{y \mid G(y)=\infty\} & =\left\{y \mid \forall n, \exists m G_{m}(y)>n\right\} \\
& =\bigcap_{n}\left\{y \mid G_{m}(y)>n\right\}
\end{aligned}
$$

is a $G_{\delta}$.

\section{§3. The Main Technical Lemma}

In this section we'll prove 
Lemma 3.1. Let $d \rho$ obey (1.2). Then

$$
\{F(y) \mid G(y)<\infty \text { and } y \in \operatorname{supp}(d \rho)\}
$$

is a countable union of nowhere dense subsets of $\mathrm{R}$.

Note that $G(y)<\infty$ implies the integral defining $F(y)$ is absolutely convergent and $F(y)$ is real. The proof will depend critically on the fact that $F$ is the boundary value of an analytic function. That such considerations must enter is seen by

Example. Let $A \subset[0,1]$ be a nowhere dense set of positive measure (e.g., remove the middle open $\frac{1}{4}$ from $[0,1]$, the middle $\frac{1}{9}$ from the remaining two pieces, the middle $\frac{1}{16} \ldots, \frac{1}{n^{2}}$ at the $(n-1)$ st step). Let

$$
\tilde{F}(y)=|A \cap[0, y]|
$$

where $|\cdot|$ is Lebesgue measure. Then $\tilde{F}$ is Lipschitz; indeed, if $x<y,|\tilde{F}(x)-\tilde{F}(y)|$ $=|A \cap[x, y]| \leq|x-y|$. But $\tilde{F}[A]=[0,|A|]$ has non-empty interior. Thus for our $F$, we need more than just Lipschitz properties (our $F$ is certainly not Lipschitz but $F \quad\{y \mid G(y)<\alpha\}$ is the restriction of a Lipschitz function to that set).

The idea of the proof will be to break up $\{y \mid G(y)<\infty, y \in \operatorname{supp}(d \rho)\}$ into a countable union of nowhere dense sets, $A_{n}$, so that $F$ is a homeomorphism on each of those sets. On each $A_{n}, G$ will be close to constant. We'll use:

Lemma 3.2. Let $B \subset \mathrm{R}$ be a nowhere dense set and let $F: B \rightarrow \mathrm{R}$ be a function obeying for $x<y$, with $x, y \in B$ :

$$
\alpha(y-x)<F(y)-F(x)<\beta(y-x)
$$

for fixed $\alpha, \beta>0$. Then $F[B]$ is nowhere dense.

Proof. By (3.1) $F$ has a unique continuum extension to $\bar{B}$ obeying (3.1). R $\backslash \bar{B}$ is a union of intervals $\left(x_{i}, y_{i}\right)$ with $x_{i}, y_{i} \in \bar{B}$. Extend $F$ to the interval by linear interpolation using slope $\frac{1}{2}(\alpha+\beta)$ on any semi-infinite subintervals of $\mathrm{R} \backslash \bar{B}$. The extended $F$ also obeys (3.1) and so defines a homeomorphism of R to R. As a homeomorphism, it takes nowhere dense sets to nowhere dense sets.

Proof of Lemma 3.1. We first break $A=\{y \in \operatorname{supp}(d \rho) \mid G(y)<\infty\}$ into a countable family of sets $A_{n}$ so that for each $n$, there is $a_{n}>0, \delta_{n}>0$ so that

(i) for $y \in A_{n}, \frac{8 a_{n}}{9}<G(y) \leq a_{n}$;

(ii) for $y \in A_{n}, \int_{|x-y|<\delta_{n}} \frac{d \rho(x)}{|x-y|^{2}} \leq \frac{a_{n}}{21}$;

(iii) $\bigcup_{y \in A_{n}}\left[y-\beta \delta_{n}, y+\beta \delta_{n}\right]$ is connected where $\beta=\frac{1}{18}\left(\frac{3}{4}\right)^{3 / 2}$.

Such a breakup exists for we can first break R into intervals $\left(\left(\frac{8}{9}\right)^{m+1},\left(\frac{8}{9}\right)^{m}\right]$ and pigeonhole $G$ by its values. Since $G(y)<\infty$ implies $\lim _{\delta \downarrow 0} \int_{|x-y|<\delta} \frac{d \rho(x)}{(x-y)^{2}}=0$, we 
can break each such set into countably many sets where (ii) holds. Then we can break each such set into countably many sets so that (iii) holds by looking for gaps of size longer than $\delta_{n} \beta$.

Each $A_{n}$ is nowhere dense by Thm. 2 and we'll show that on $A_{n}, y>x$ implies that

$$
\frac{1}{3} a_{n}(y-x)<F(y)-F(x)<\frac{5}{3} a_{n}(y-x)
$$

so that the lemma follows from Lemma 3.2.

Define $\ell_{n}=\beta \delta_{n}$ and $\epsilon_{n}=\sqrt{\frac{3}{4}} \ell_{n}$. For $y \in A_{n}$, let $\triangle_{n}(y)$ be the triangle in C (see Fig. 3.1):

$$
\triangle_{n}(y)=\left\{z\left|0<\operatorname{Im} z \leq \epsilon_{n},\right| \arg (z-y)-\frac{\pi}{2} \mid \leq \frac{\pi}{6}\right\} .
$$

This is the equilateral triangle of side $\ell_{n}$ with one side parallel to the real axis at distance $\epsilon_{n}$ from that axis and the opposite vertex at $y$.

For $z \in \triangle_{n}(y)$, define

$$
G(z)=\int \frac{d \rho(x)}{(x-z)^{2}}=\frac{d F}{d z}
$$

We claim that for $z \in \triangle_{n}$

$$
\left|G(z)-a_{n}\right| \leq \frac{a_{n}}{3} .
$$

Accepting (3.3) for the moment, let us prove (3.2). By the fundamental theorem of calculus, (3.3) implies for $z, z^{\prime} \in \triangle_{n}(y)$ :

$$
\left|F(z)-F\left(z^{\prime}\right)-a_{n}\left(z-z^{\prime}\right)\right| \leq \frac{a_{n}}{3}\left|z-z^{\prime}\right| .
$$

By hypothesis (iii) on $A_{n}, \bigcup_{y \in A_{n}} \triangle_{n}(y)$ is connected and so, given $y<y^{\prime} \in A_{n}$, we can find a finite sequence $y_{0}=y<y_{1}<\cdots<y_{n}=y^{\prime}$ and $z_{1}, \ldots, z_{n}$ so that (see Fig. 3.2)

$$
z_{j} \in \triangle_{n}\left(y_{j-1}\right) \cap \triangle_{n}\left(y_{j}\right) \quad \text { and } \quad\left|z_{j}-y_{j-1}\right|=\left|z_{j}-y_{j}\right|=\left|y_{j}-y_{j-1}\right| .
$$

By (3.4) and (3.5)

$$
\left|F(y)-F\left(y^{\prime}\right)-a_{n}\left(y-y^{\prime}\right)\right| \leq \frac{2 a_{n}}{3}\left(y-y^{\prime}\right)
$$

which is $(3.2)$.

Thus we need only prove (3.3) We write

$$
\left|G(z)-a_{n}\right| \leq\left|b_{0}\right|+\left|b_{1}\right|+\left|b_{2}\right|+\left|b_{3}\right|
$$


where

$$
\begin{aligned}
& b_{0}=G(y)-a_{n} \\
& b_{1}=\int_{|x-y|<\delta_{n}} \frac{d \rho(x)}{|x-z|^{2}} \\
& b_{2}=\int_{|x-y|<\delta_{n}} \frac{d \rho(x)}{(x-y)^{2}} \\
& b_{3}=\tilde{G}(z)-\tilde{G}(y)
\end{aligned}
$$

with

$$
\tilde{G}(z)=\int_{|x-y| \geq \delta_{n}} \frac{d \rho(x)}{(x-z)^{2}} .
$$

By hypothesis (i) on $A_{n},\left|b_{0}\right| \leq \frac{a_{n}}{9}$.

By hypothesis (ii) on $A_{n},\left|b_{2}\right| \leq \frac{a_{n}}{21}$. By elementary trigonometry,

$$
z \in \triangle(y) \quad \text { and } \quad x \in \mathrm{R} \Rightarrow|z-y| \geq \sqrt{\frac{3}{4}}|x-y| .
$$

Thus

so

$$
\left|b_{1}\right| \leq \frac{4}{3}\left|b_{2}\right|
$$

$$
\left|b_{1}\right|+\left|b_{2}\right| \leq \frac{7}{3} \frac{a_{n}}{21}=\frac{a_{n}}{9} .
$$

Finally, using the fundamental theorem of calculus and (3.6)

$$
\begin{aligned}
\left|b_{3}\right| & \leq 2|z-y|\left(\frac{4}{3}\right)^{3 / 2} \delta_{n}^{-1} \int_{|x-y| \geq \delta_{n}} \frac{d \rho(x)}{(x-y)^{2}} \\
& \leq 2 \delta_{n}^{-1} \ell_{n}\left(\frac{4}{3}\right)^{3 / 2} a_{n} \\
& =2 \beta\left(\frac{4}{3}\right)^{3 / 2} a_{n}=\frac{a_{n}}{9}
\end{aligned}
$$

by definition of the constant $\beta$. Thus (3.3) holds.

\section{$\S 4$. Proof of the Main Theorem}

Our goal here is to prove Thm. 3 (from §1) and derive some simple abstract consequences of it.

Proof of Thm. 3. The maps $M_{1}: \mathrm{R} \backslash\{0\} \rightarrow \mathrm{R} \backslash\{0\}$ by $M_{1}(\lambda)=-\lambda^{-1}$ and $M_{2}:[0, \pi) \rightarrow \mathrm{R} \cup\{\infty\}$ by $M_{2}(\theta)=\cot \left(\theta-\theta_{0}\right)$ are homeomorphisms. Thus, by Lemma 3.1,

$$
\left\{\lambda \mid \exists E \text { s.t. } G(E)<\infty, E \in \operatorname{spec}\left(A_{0}\right), F(E)=-\lambda^{-1}\right\}
$$


and

$$
\left\{\theta \mid \exists E \text { s.t. } G(E)<\infty, E \in \operatorname{spec}\left(A_{0}\right), F(E)=\cot \left(\theta-\theta_{1}\right)\right\}
$$

are countable unions of nowhere dense sets. Its complement is thus a dense set by the Baire category theorem. But by Thm. 1, this is precisely $\left\{\lambda \mid A_{\lambda}\right.$ has no eigenvalues on $\left.\operatorname{spec}\left(A_{0}\right)\right\}$, which we conclude is dense. By general principles [12], it is also a $G_{\delta}$.

Here are some simple corollaries of Thm. 3. We state them in the rank one case but they hold in the $\cot \left(\theta-\theta_{0}\right)$ B.C. case also.

Corollary 4.1. Suppose that $A_{0}$ is an operator with no a.c. spectrum and $P$ is a rank one projection whose range is cyclic for $A$. Then for a dense $G_{\delta}$ of $\lambda$ 's, $A_{\lambda}=A+\lambda P$ has only singular continuous spectrum in $\operatorname{spec}\left(A_{0}\right)^{\text {int }}$.

Proof. $A_{\lambda}$ has no a.c. spectrum since the a.c. spectrum is left invariant by finite rank perturbations. $\operatorname{spec}\left(A_{0}\right)$ has no eigenvalues for a dense $G_{\delta}$ of $\lambda$. There can be eigenvalues on $\mathrm{R} \backslash \operatorname{spec}\left(A_{0}\right)$ and so point spectrum on $\partial\left(\operatorname{spec}\left(A_{0}\right)\right)$. But there cannot be point spectrum in $\operatorname{spec}\left(A_{0}\right)^{\text {int }}$.

Corollary 4.2. Suppose that $A_{0}$ is an operator with no a.c. spectrum and an interval $[\alpha, \beta] \subset \operatorname{spec}\left(A_{0}\right)$. Let $P$ be a rank one projection whose range is a cyclic vector for $A_{0}$. Then for a dense $G_{\delta}$ of $\lambda$ 's, $A_{0}+\lambda P$ has singular continuous spectrum on all of $(\alpha, \beta)$ and only singular continuous spectrum there.

Proof. A direct consequence of Cor. 4.1.

\section{$\S 5$. Examples and Consequences}

Our first class of examples are half-line Schrödinger operators:

Theorem 5.1. Let $V(x)$ be a locally $L_{1}$ function on $[0, \infty)$ and let $H_{\theta}=-\frac{d^{2}}{d x^{2}}+$ $V(x)$ with $\theta$ boundary conditions. Suppose there is some $\theta_{0}$ and $\alpha<\beta$ so that

(i) $[\alpha, \beta] \subset \operatorname{spec}\left(H_{\theta_{0}}\right)$.

(ii) for Lebesgue a.e., $E_{0} \in[\alpha, \beta]$, there exists a function $\varphi_{E_{0}}$ obeying

$$
\begin{gathered}
-\varphi^{\prime \prime}(x)+V(x) \varphi(x)=E_{0} \varphi(x) \\
\int_{0}^{\infty}|\varphi(x)|^{2} d x<\infty .
\end{gathered}
$$

Then:

(a) For a dense $G_{\delta}$ of $E$ 's in $[\alpha, \beta]$, there is no solution of (5.1) obeying (5.2).

(b) For Lebesgue a.e. $\theta, H_{\theta}$ has only point spectrum in $(\alpha, \beta)$.

(c) For a dense $G_{\delta}$ of $\theta, H_{\theta}$ has only singular continuous spectrum in $(\alpha, \beta)$.

Remarks. 1. (b), (c) say that there are disjoint sets both locally uncountable where $H_{\theta}$ shifts between purely pure point and purely singular continuous spectrum on $[\alpha, \beta]$.

2. It may well happen that there are $\theta$ 's with spectrum of mixed type. 
3. For precursors of (a), see del Rio [4].

Proof. If $E$ is such that (5.1) has a solution obeying (5.2), then $\varphi_{E}$ obeys some boundary condition at $x=0$ and so $E$ is an eigenvalue of some $H_{\theta}$. Thus (a) follows from Thm. 2 .

To prove (b), note that if $E_{0}$ has a solution obeying (5.1-2) and $E_{0}$ is not an eigenvalue of $H_{\theta_{0}}$, then $\lim _{\epsilon \downarrow 0} \int_{0}^{\infty}|G(0, x ; E+i \epsilon)|^{2} d x<\infty$. Now apply the ideas of Kotani [11] and Simon-Wolff [13].

(c) follows from Thm. 3 .

Example 5.2. Suppose that $[a, b] \subset \operatorname{spec}\left(-\frac{d^{2}}{d x^{2}}+V(x)\right)$ and that for a.e. $E \subset$ $[a, b], \lim _{x \rightarrow \infty} \frac{1}{|x|} \ln \left\|T_{E}(x)\right\|=\gamma(E)$ and is positive. Here $T$ is the standard transfer matrix, that is,

$$
T_{E}(x)=\left(\begin{array}{ll}
\varphi_{1}(x) & \varphi_{2}(x) \\
\varphi_{1}^{\prime}(x) & \varphi_{2}^{\prime}(x)
\end{array}\right)
$$

where $\varphi_{i}$ obeys $-u^{\prime \prime}+V u=E u$ with $\varphi_{1}(0)=\varphi_{2}^{\prime}(0)=1$ and $\varphi_{1}^{\prime}(0)=\varphi_{2}(0)=0$. Then (a) implies there must be a dense $G_{\delta}$ of $E$ where either $\lim \frac{1}{|x|}\left\|T_{E}(x)\right\|$ fails to exist or is zero. Thus, a positive limit can never exist for all $E$ in an interval. Results of this genre have been found previously by Goldsheid [6] and Carmona [2].

Example 5.3. Consider a one-dimensional random model with localization, for example, the GMP model $[7,2]$. Then for almost every $E$ in $[\alpha, \infty)$, one knows $\gamma(E)$ exists and is positive. It follows from Thm. 5.1 that for a locally uncountable set of boundary conditions (a Lebesgue typical set), one has pure point spectrum, while for a distinct set of locally uncountable boundary conditions (a Baire typical set), one has singular spectrum. Each spectral type is unstable to change to the other spectral type.

Example 5.4. Let $H=-\frac{d^{2}}{d x^{2}}+\cos (\sqrt{x})$ on $L^{2}(0, \infty)$, a model studied by Stolz [14]. As proven by him for any boundary condition $\theta$ :

$$
\operatorname{spec}\left(H_{\theta}\right)=[-1, \infty)
$$

$\operatorname{spec}\left(H_{\theta}\right)$ is purely absolutely continuous on $(1, \infty)$. Kirsch et al. [10] prove that for a.e. $\theta, H_{\theta}$ has pure point spectrum in $[-1,1]$ only. Our results show that for a dense $G_{\delta}$ of $\theta$, the spectrum is purely singular continuous. Once again you have intertwined purely pure point and purely singular continuous spectrum.

Finally, we consider the case of the Anderson model:

Theorem 5.5. Let $V_{\omega}$ be a $\nu$-dimensional model with uniform distribution on $[a, b]$ at each set. Suppose that the corresponding Jacobi matrix $h_{\omega}$ has point spectrum in $[\alpha, \beta]$ for a.e. $V_{\omega}$. Fix $V_{\omega}(n)$ typical. Then for a.e., choice $V_{\omega}(0) \in[a, b], h_{\omega}$ has pure point spectrum in $[\alpha, \beta]$; but for a dense $G_{\delta}$ of values of $V_{\omega}(0), h_{\omega}$ has purely singular spectrum in $[\alpha, \beta]$.

This follows from Thm. 3 . 
Acknowledgment. We would like to thank H. Kalf for useful conversations.

\section{REFERENCES}

[1] N. Aronszajn, On a problem of Weyl in the theory of singular Sturm-Liouville equations, Am. J. Math. 79 (1957), 597-610.

[2] R. Carmona, Exponential localization in one-dimensional disordered systems, Duke Math. J. 49 (1982), 191-213.

[3] R. Carmona and J. Lacroix, Spectral theory of random Schrödinger operators, Birkhäuser, Boston, 1990.

[4] R. del Rio, A forbidden set for embedded eigenvalues (to appear Proc. AMS).

[5] W. Donoghue, On the perturbation of spectra, Comm. Pure App. Math. 18 (1965), 559-579.

[6] I. Goldsheid, Asymptotics of the product of random matrices depending on a parameter, Soviet Math. Dokl. 16 (1975), 1375-1379.

[7] I. Goldsheid, S. Molchanov and L. Pastur, A pure point spectrum of the stochastic onedimensional Schrödinger equation, Func. Anal. Appl. 11 (1977), 1-10.

[8] A. Gordon, On exceptional value of the boundary phase for the Schrödinger equation of a half-line, Russian Math Surveys 47 (1992), 260-261.

[9] localization, preprint.

[10] W. Kirsch, S. Molchanov and L. Pastur, One dimensional Schrödinger operators with high potential barriers, Operator Theory: Advances and Applications, vol. 57, Birkhäuser Verlag, Basel, 1992, pp. 163-170.

[11] S. Kotani, Lyapunov exponents and spectra for one-dimensional random Schrödinger operators, Contemp. Math. 50 (1986), 277-286.

[12] B. Simon, Operators with singular continuous spectrum, I. General operators, preprint.

[13] B. Simon and T. Wolff, Singular continuous spectrum under rank one perturbations and localization for random Hamiltonians, Comm. Pure Appl. Math. 39 (1986), 75-90.

[14] G. Stolz, Bounded solutions and absolute continuity of Sturm-Liouville operators, J. Math. Anal. Appl. 169 (1992), 210-228. 